\title{
Plattform-Bereitstellungsstrategien im Maschinen- und Anlagenbau: Strategien deutscher Unternehmen im Industrie 4.0-Kontext
}

\author{
Benedict Bender (D) $\cdot$ Sander Lass $\cdot$ Natalie Habib $\cdot$ Laura Scheel
}

Eingegangen: 3. April 2020 / Angenommen: 25. Juli 2020 / Online publiziert: 11. August 2020

(C) Der/die Autor(en) 2020

Zusammenfassung Digitale Plattformen finden zunehmende Verbreitung in unterschiedlichen Industriezweigen. Immer mehr Unternehmen sind an der Erschließung verbundener Potenziale für ihr Geschäft interessiert. Im Maschinen- und Anlagenbau wird die Vernetzung von Maschinen zunehmend ein Wettbewerbsfaktor für Hersteller. Der Einsatz digitaler Plattformen im Maschinen- und Anlagenbau bietet Herstellern Möglichkeiten zur gezielten Erweiterung des Geschäftsmodells. Für die Bereitstellung digitaler Plattformen können Unternehmen auf unterschiedliche Strategien zurückgreifen. Hierbei sollten Unternehmen die für ihre Konstellation geeignete Variante systematisch identifizieren, um die angestrebten Ziele zu erreichen. Die geeignete Strategie ist von einer Vielzahl an Faktoren abhängig. Als Grundlage für die Identifikation der geeigneten Strategie bietet dieser Beitrag eine systematische Untersuchung der möglichen Bereitstellungsstrategien für Unternehmen. Neben der theoretischen Systematisierung werden gegenwärtig genutzte Strategien am Beispiel des Maschinen- und Anlagenbaus in Deutschland vorgestellt. Zudem werden spezifische Merkmale, welche die Nutzung einer Strategie beeinflussen, als Ansatzpunkt für einen Strategieformulierungsprozess identifiziert. Im Maschinenund Anlagenbau ist die Bereitstellung einer eigenen Plattform, insbesondere bei

\footnotetext{
B. Bender $(\bowtie) \cdot$ S. Lass $\cdot$ N. Habib $\cdot$ L. Scheel Lehrstuhl für Wirtschaftsinformatik, insb. Prozesse und Systeme, Universität Potsdam, August-Bebel-Str. 89, 14482 Potsdam, Deutschland

E-Mail: benedict.bender@wi.uni-potsdam.de

S. Lass

E-Mail: sander.lass@wi.uni-potsdam.de

N. Habib

E-Mail: natalie.habib@wi.uni-potsdam.de

L. Scheel

E-Mail: laura.scheel@wi.uni-potsdam.de
} 
Großunternehmen vorherrschend. Die Strategien von KMU unterschieden sich von Großunternehmen.

Schlüsselwörter Digitale Plattformen · KMU · Maschinen- und Anlagenbau • Industrie $4.0 \cdot$ Plattform-Bereitstellungsstrategien

\title{
Platform Delivery Strategies in Mechanical and Plant Engineering: Strategies of German Companies in the Industry 4.0 Context
}

\begin{abstract}
Digital platforms are becoming increasingly widespread across different industries. More and more companies are interested in developing related potential for their business. In mechanical and plant engineering, the networking of machines becomes increasingly important and a strategic advantage for manufacturers. The use of digital platforms in mechanical and plant engineering offers manufacturers opportunities for targeted expansion of their business model. For the provision of digital platforms, companies can use different strategical approaches. Companies should systematically identify the variant suitable for their constellation in order to achieve the desired objectives. The appropriate strategy depends on a variety of factors. As a basis for the identification of the appropriate strategy, this article offers a systematic overview of the possible deployment strategies for companies. In addition to the theoretical systematization, currently used strategies are presented using the example of the mechanical and plant engineering industry in Germany. In addition, specific features that influence the use of a strategy are identified as a starting point for a strategy formulation process. In mechanical and plant engineering, the provision of an own platform is predominant, especially in large companies. The strategies of SMEs differ from those of large companies.
\end{abstract}

Keywords Digital platforms · SME · Machinery and plant engineering · Industry $4.0 \cdot$ Platform delivery strategies

\section{Einleitung}

Digitale Plattformen finden zunehmende Verbreitung in zahlreichen Industriezweigen. Die wachsende Bedeutung digitaler Plattformen zeichnet sich im Endkundenbereich ebenso wie im professionellen Bereich ab. Immer mehr Unternehmen und ganze Industrien sind interessiert, mögliche Potenziale zu erschließen. Die zunehmende Digitalisierung von Produkten und deren Vernetzung stellen notwendige Voraussetzungen für die Erschließung dieser Potenziale dar.

Auch der Maschinen- und Anlagenbau profitiert von der Vernetzung der Maschinen und Anlagen (VDMA 2018). Im Kontext von Industrie 4.0 kommt der Vernetzung von Maschinen und den damit verbundenen Möglichkeiten zur direkten Kommunikation und Interaktion (M2M) eine gesteigerte Bedeutung zu (Gronau et al. 2016). Die Vernetzung der Maschinen wird zunehmend ein Wettbewerbsfaktor für Hersteller, welcher wiederum die Grundlage für die Erschließung von Potenzialen bei den Anwendern darstellt (Weinert et al. 2017). Die Vernetzung der Komponen- 
ten kann dabei auf unterschiedlichen Ebenen erfolgen. Neben der Vernetzung direkt physisch benachbarter Maschinen (z.B. innerhalb eines Werkes), können Maschinen standortübergreifend innerhalb eines logischen Kontexts (z. B. innerhalb eines Firmennetzes) bis zur direkten Anbindung an das Internet (z.B. zur Einbindung in die gesamte Supply-Chain) erfolgen. Während die Verbindung von Maschinen zur direkten Steuerung zumeist mittels Automatisierungsansätzen lokal realisiert ist, verfolgen erste Ansätze bereits standortübergreifende Konzepte zur Koordination (Bender et al. 2019). Der Einsatz von digitalen Plattformen zur Anbindung von Maschinen, welcher zumeist mit der Nutzung von Cloud-Infrastrukturen einhergeht, bietet durch die globale Erreichbarkeit der Maschinen und der Plattform neuartige Potenziale (Dremel und Herterich 2016).

Die Potenziale digitaler Plattformen für den Maschinen- und Anlagenbau sind vielfältig. Beispielsweise können durch den Einsatz digitaler Plattformen erweiterte Funktionen und zusätzliche Services angeboten werden. Konzepte wie die zustandsbasierte Wartung profitieren von der Anbindung der Maschinen. Die Bereitstellung von aktuellen Zustandsinformationen und detaillierten Fehlerdiagnosen ermöglicht einerseits die situationsgerechte Behebung sowie andererseits die Chance, möglichen Betriebsausfällen vorzubeugen (Predictive Maintainance) (Mobley 2002). Die gezielte Erweiterung des Geschäftsmodells ist im Maschinen- und Anlagenbau ein Interesse vieler Anbieter (Demont und Paulus-Rohmer 2017; Kampker et al. 2017; Pflaum und Schulz 2018).

Wenngleich der Einsatz digitaler Plattformen umfangreiche Potenziale bietet, ist ihre Bereitstellung für Unternehmen mit zahlreichen Herausforderungen verbunden (VDMA 2018). Dies gilt für KMU im Besonderen (Bender et al. 2020; Bollhöfer et al. 2016). Unter anderem sind spezialisiertes Know-how sowie entsprechende Engineering- und Entwicklungskapazitäten erforderlich. Aufgrund der Vielzahl an verfügbaren Plattformen ist von einer langfristigen Konsolidierung auszugehen (VDMA 2018). Aufgrund des Wettbewerbsdrucks sind Anbieter von Maschinen und Anlagen gefordert, Plattformen mit ausreichender Differenzierung anzubieten, um entsprechende Wettbewerbsvorteile realisieren zu können. Für die Bereitstellung einer digitalen Plattform existieren unterschiedliche Möglichkeiten. Unternehmen sollten die für ihre individuelle Konstellation geeignete Variante systematisch identifizieren, um die angestrebten Ziele zu erreichen. Die ideale Form der Bereitstellung einer digitalen Plattform ist von einer Vielzahl an Faktoren abhängig. Als Grundlage für die Identifikation der geeigneten Strategie bietet dieser Beitrag eine systematische Untersuchung der möglichen Bereitstellungsstrategien für Unternehmen. Neben der theoretischen Systematisierung sollen die gegenwärtig genutzten Strategien systematisch am Beispiel des Maschinen- und Anlagenbaus erhoben werden. Es wird dementsprechend untersucht:

FF1: Welche Strategien nutzen Unternehmen (des Maschinen- und Anlagenbaus) zur Bereitstellung digitaler Plattformen für Ihre Kunden?

Die Ermittlung von Einflussfaktoren auf die Auswahl der unterschiedlichen Bereitstellungsstrategien wird als Grundlage für den Strategieformulierungsprozess untersucht. Am Beispiel des Maschinen- und Anlagenbaus wird daher untersucht: 
FF2: Welche Faktoren differenzieren die Nutzung der gewählten Bereitstellungsstrategien für digitale Plattformen?

Der Fokus des Beitrags liegt dabei auf der Bereitstellung einer digitalen Plattform für Kunden des Unternehmens. Davon zu differenzieren ist die Nutzung einer digitalen Plattform durch Unternehmen des Maschinen- und Anlagenbaus (z. B. zur Überwachung der eigenen Fertigungsprozesse). Während sich die Nutzung vorwiegend auf interne Prozesse des Unternehmens fokussiert, so bezieht sich die Bereitstellung einer Plattform auf das Angebot des Unternehmens an Kunden.

\section{Strategien zur Bereitstellung digitaler Plattformen}

Während das Angebot und die Potenziale digitaler Plattformen bereits Berücksichtigung in unterschiedlichen Untersuchungen gefunden haben (Dremel und Herterich 2016; Oberländer et al. 2019; Pflaum und Schulz 2018), so bleiben die strategischen Möglichkeiten für die Bereitstellung einer Plattform durch Unternehmen weitestgehend unberücksichtigt. Insbesondere für Unternehmen ergeben sich weitreichende Unterschiede je nach Strategie der Bereitstellung. Während Unternehmen beispielsweise bei einer eigenen Plattform vollständige Freiheit in der Gestaltung und Realisierung haben, so sind diese bei der Anbindung und Nutzung von externen Plattformen weitestgehend an die gegebenen Funktionen gebunden (Bender et al. 2020). Insbesondere für die Engineering-fokussierten Unternehmen des Maschinenund Anlagenbaus stellt die vollständige Eigenentwicklung einer digitalen Plattform als komplexes IT-Projekt eine große Herausforderung dar.

Die Systematisierung unterschiedlicher Bereitstellungsstrategien ermöglicht die Bewertung der Alternativen für Unternehmen und der sich daraus ergebenden Implikationen. Die Systematisierung stellt somit eine wesentliche Grundlage für die gezielte Festlegung der geeigneten Strategie und die Erreichung der damit angestrebten Ziele dar.

Dieser Beitrag adressiert die bisherige Forschungslücke im Hinblick auf die fehlende Systematisierung, indem mögliche Bereitstellungsstrategien aufgezeigt werden. Die Erarbeitung der Systematisierung basiert auf der Analyse einer Vielzahl von Referenzfällen sowie der zur Realisierung verfügbaren technischen Möglichkeiten für eine Plattform. Der folgende Abschnitt differenziert mögliche Bereitstellungsstrategien und ergänzt diese durch Realbeispiele. Die Systematisierung bildet damit das erste Ergebnis des Beitrags sowie die Grundlage für die Analyse im Hinblick auf die erste Forschungsfrage.

Abb. 1 bietet eine Systematisierung möglicher Bereitstellungstrategien für digitale Plattformen durch Unternehmen. Wesentliches Differenzierungskriterium ist der Grad an Eigenständigkeit und den damit verbundenen Gestaltungsmöglichkeiten des anbietenden Unternehmens, welches die Grundlage für die Gestaltung von Angeboten für die Kunden beeinflusst. Unternehmen haben die Möglichkeit eine Plattform selbst bereitzustellen (Strategien 1, 2, 3), diese zusammen mit weiteren Unternehmen anzubieten (Strategie 4) oder vollständig durch Dritte bereitstellen zu lassen (Strategie 5). Das Angebot einer eigenen Plattform kann auf unterschiedliche Weisen erfolgen. 

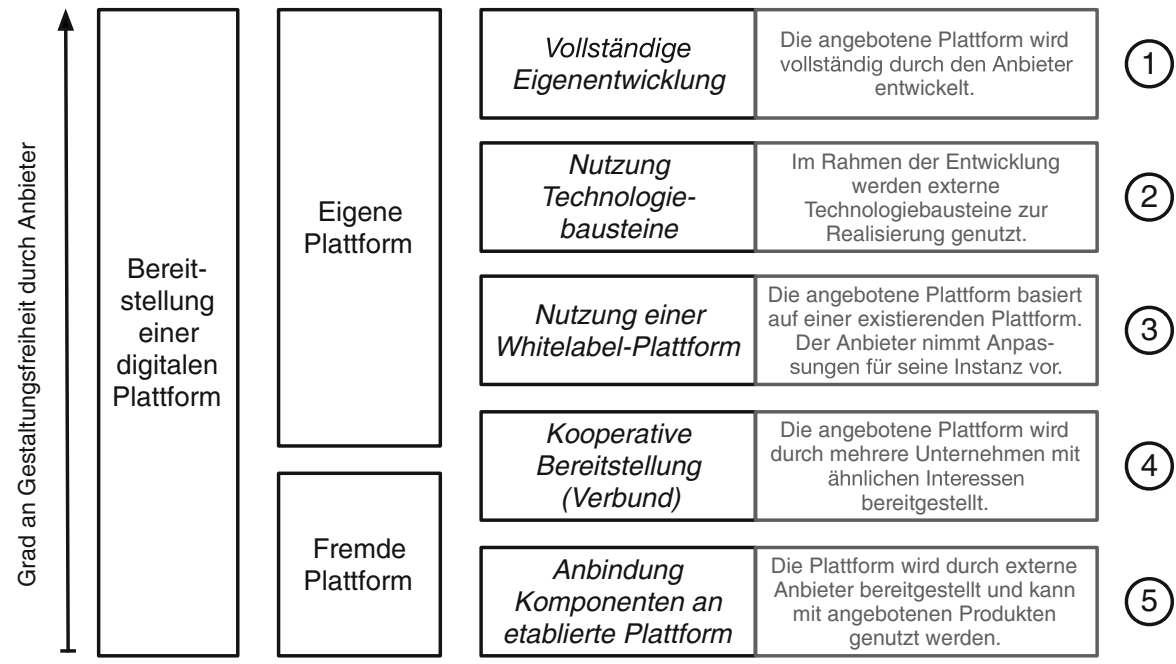

Abb. 1 Übersicht Strategien zur Bereitstellung einer Digitalen Plattform

Strategie 1: bezeichnet die vollständige Eigenentwicklung einer digitalen Plattform durch das anbietende Unternehmen. Sämtliche Softwarekomponenten werden hierbei vollständig durch das Unternehmen selbst entwickelt. Ein Beispiel hierfür stellt ein Spezialhersteller von Oberflächenbearbeitungsanlagen und Fördertechnik dar. Das mittelständische Unternehmen bietet eine vollständig selbst entwickelte Plattform an. Die Plattform stellt Kunden ein Dashboard zur Verfügung, das mithilfe von cloudbasierten Datenbanken die Auswertung von Betriebsdaten entlang des kompletten Produktionsablaufs auf verschiedenen Endgeräten ermöglicht.

Strategie 2: Nutzung Technologiebausteine beschreibt die Bereitstellung einer eigenen Plattform unter Nutzung von existierenden Technologiebausteinen. Die Plattform wird dabei im Wesentlichen selbst realisiert, hierfür werden etablierte Komponenten eingesetzt. Die Abgrenzung zwischen Strategie 1 und 2 bedarf der genauen Spezifikation. Insbesondere die Festlegung ab wann ein eingesetztes Fragment ein Technologiebaustein darstellt. Als Beispiel für Strategie 2 kann ein Hersteller von Werkzeugmaschinen angeführt werden, der eine individuelle Plattform anbietet, welche die Maschinendaten der Kunden über die Microsoft Azure Cloud kommuniziert und bereitstellt. Dabei kann die Cloud als externer Technologiebaustein betrachtet werden, insbesondere wenn diese neben der technischen Cloudinfrastruktur (IaaS) bereits Plattformsoftware in Form von Modulen (z. B. Datenverwaltung, Schnittstellen oder Funktionen) bereitstellt. Die Maschinen dieses Großunternehmens sind mit einer speziellen Software ausgestattet, um die Anbindung an die Plattform zu gewährleisten. Die Plattform bietet Funktionen für die Datenanalyse zur Minimierung der Ausschussrate, die vorausschauende Wartung von Maschinen und beinhaltet zudem einen integrierten Shop für Ersatzteile. 
Strategie 3: Nutzung einer Whitelabel-Plattform beschreibt das Angebot einer eigenen digitalen Plattform unter Verwendung externer Plattformtechnologien, welche zu einem gewissen Grad angepasst werden können. Zumeist kann das Frontend der Plattform an die Corporate Identity des anbietenden Unternehmens angepasst werden. In Abhängigkeit von der Ausgestaltung des Angebots können z. B. spezialisierte Funktionen angeboten werden. Die digitale Plattform eines Großunternehmens aus der Werkzeugmaschinenbranche stellt ein Beispiel für diese Strategie dar. Dabei nutzt das Unternehmen das Produkt SAP Leonardo als Whitelabel-Plattform, wobei Funktionen und Auftreten der Plattform angepasst wurden. Mithilfe dieser digitalen Plattform können die Maschinen und Anlagen der Kunden des Maschinenherstellers über eine Cloud vernetzt werden und so die Realisierung eines Smart Warehouse unterstützen.

Strategie 4: $\quad$ Kooperative Bereitstellung bezeichnet einen hybriden Ansatz zur Bereitstellung einer digitalen Plattform, wobei weitere Unternehmen z.B. in einem Verbund an der gemeinsamen Bereitstellung einer Plattform beteiligt sind. Eine Plattform kann durch mehrere Unternehmen entwickelt und gemeinsam angeboten werden. Als Beispiel für eine kooperative Bereitstellung kann eine Plattform aus dem Bereich der Landwirtschaft herangezogen werden. Der herstellerübergreifende Plattformverbund ermöglicht die Anbindung unterschiedlicher Agrarmaschinen. Mit der offenen Plattform, welche gemeinsam mit über 40 Partnern aus der Landwirtschaft entwickelt wurde, können unterschiedliche Anlagen und Applikationen gekoppelt werden. Spezialisierte Funktionen unterstützen Landwirte gezielt bei ihrer Arbeit.

Die Bereitstellung einer Plattform bzw. der zugehörigen Funktionen kann auch mittels einer vollständig externen Plattform erfolgen.

Strategie 5: Anbindung an etablierte Plattform bezeichnet die Anbindung der angebotenen Komponenten an eine unabhängige Plattform, welche durch Dritte bereitgestellt wird. Bei dieser Strategie hat das Unternehmen geringen bis keinen Einfluss auf die Plattform. Ein mittelständisches Unternehmen aus dem Bereich der Drucklufttechnik stellt ein Beispiel für die Anbindung an eine etablierte Plattform dar. Der Maschinenhersteller nutzt die Cloud der Dinge der Telekom und bietet seinen Kunden diese unabhängige Lösung als Produkt zur Überwachung der Druckluftanlagen, vorausschauenden Wartung und einer erhöhten Ausfallsicherheit an. Im Rahmen dieser Strategie können Hersteller auch die Anbindung an unterschiedliche Plattformen ermöglichen.

\section{Methodik}

Dieser Abschnitt stellt die für die Beantwortung der Forschungsfragen und dem damit verbundenen Erkenntnisinteresse angewendeten Methodik dar. Zur Ermittlung der eingesetzten Strategien (Forschungsfrage 1) sowie entsprechenden Differenzierungsmerkmalen (Forschungsfrage 2) ist eine systematische Erhebung notwendig. Im Rahmen der Recherche konnten keine Forschungsarbeiten identifiziert werden, 
die die Formen der Bereitstellungsstrategien systematisch erhoben haben. Dieser Beitrag strebt an, diese Forschungslücke durch eine repräsentative Erhebung zu schließen. Die Systematisierung von Bereitstellungsstrategien kann als Framework für die Analyse sowie Bewertung des gegenwärtigen Standes im Hinblick auf die genutzten Bereitstellungsstrategien genutzt werden.

\subsection{Fokus: Maschinen- und Anlagenbau}

Um ausreichend spezifische Erkenntnisse generieren zu können, wird ein konkretes Betrachtungsszenario festgelegt. Das gewählte Szenario entstammt dem Maschinenund Anlagenbau. Ein etablierter Anbieter von Industriemaschinen möchte seinen Kunden eine digitale Plattform anbieten. Die angebotenen Maschinen sind bereits internetfähig. Die Plattform soll zur Anbindung der Maschinen und darauf basierend für das Angebot von Zusatz- und Mehrwertdiensten genutzt werden. Der Anbieter steht vor der Frage, welche Bereitstellungsstrategien zur Verfügung stehen und welche für seine Situation geeignet ist. Gerade in Deutschland ist eine Vielzahl von kleinen, mittleren ebenso wie großen Unternehmen in diesem Sektor mit innovativen Technologien aktiv. Wenngleich die Voraussetzungen und Möglichkeiten nach Unternehmensgröße variieren, so sind die theoretischen Optionen für die Unternehmen gleich.

\subsection{Referenzdatenbank}

Zur Beantwortung der Forschungsfragen ist eine systematische Erhebung der relevanten Parameter im Markt erforderlich. Die Ergebnisse der Marktrecherche werden im Rahmen einer Referenzprojektdatenbank zusammengefasst. Die Datenbank stellt die Grundlage für die weitere Analyse der Ergebnisse dar. Die Marktrecherche ist vor dem Hintergrund der Reproduzierbarkeit und Vergleichbarkeit zu standardisieren. Dafür wurden Vorgaben für die Recherche und Qualifizierung der Fälle erarbeitet. Abb. 2 stellt das Vorgehen überblicksartig dar.

\subsubsection{Marktrecherche}

Die Identifikation der Referenzfälle erfolgte durch Onlinerecherche. Für die Identifikation potenzieller Plattformen wurde das Branchenverzeichnis für den Maschinenund Anlagenbau des VDMA als Grundlage genutzt. Ergänzend wurden branchenspezifische Statistiken zur Ermittlung infrage kommender Anbieter genutzt. Weiterhin wurden Fachmedien wie Zeitschriften und Messeverzeichnisse (z. B. der Hannover Messe Industrie) neben der stichwortbasierten Onlinerecherche genutzt. Im Rahmen der Recherche wurden insgesamt 181 potenzielle Fälle ermittelt.

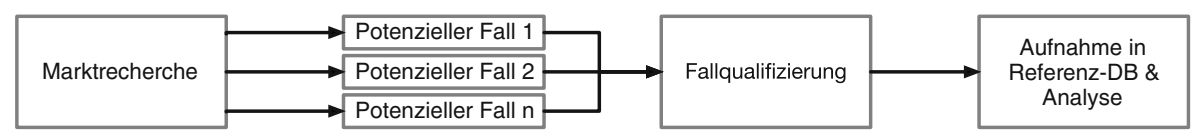

Abb. 2 Überblick Vorgehen Referenzdatenbank 
Tab. 1 Kriterien für die Qualifizierung von Referenzprojekten

\begin{tabular}{|c|c|c|}
\hline Kriterium & Spezifikation/Anforderung & Hintergrund \\
\hline Hersteller & $\begin{array}{l}\text { Der Anbieter der Plattform ist ein Hersteller } \\
\text { bzw. Anbieter von Komponenten (Maschi- } \\
\text { nen/Anlagen). Während die Herstellung eben- } \\
\text { falls durch externe Partner erfolgen kann, ist } \\
\text { entscheidend, dass der betrachtete Hersteller/ } \\
\text { Anbieter die Maschine konzeptioniert und ent- } \\
\text { wickelt hat }\end{array}$ & $\begin{array}{l}\text { Mittels dieses Kriteriums } \\
\text { soll sichergestellt werden, } \\
\text { dass der Anbieter auch } \\
\text { konzeptionellen Einfluss auf } \\
\text { die Gestaltung der Maschine } \\
\text { und Plattform hat }\end{array}$ \\
\hline Konnektivität & $\begin{array}{l}\text { Die angebotene Komponente kann direkt per } \\
\text { Internet mit der Plattform verbunden werden. } \\
\text { Für die Anbindung sind keine zusätzlichen } \\
\text { Komponenten wie Gateways o. ä. zwingend } \\
\text { notwendig }\end{array}$ & $\begin{array}{l}\text { Der Aspekt der direkten } \\
\text { Konnektivität dient der Ab- } \\
\text { grenzung von mehrstufigen } \\
\text { Anbindungs- und Steue- } \\
\text { rungskonzepten }\end{array}$ \\
\hline Eigenständigkeit & $\begin{array}{l}\text { Die angebotene Komponente (Maschine/ } \\
\text { Anlage) funktioniert in Kombination mit der } \\
\text { Plattform eigenständig. Es sind keine zusätz- } \\
\text { lichen Steuerungskomponenten auf Soft- und } \\
\text { Hardwareebene für die Nutzung der Plattform- } \\
\text { funktionen erforderlich }\end{array}$ & $\begin{array}{l}\text { Die Anbindung sollte ohne } \\
\text { Zusatzkomponenten möglich } \\
\text { sein, um dem Aspekt der } \\
\text { direkten Anbindung von } \\
\text { Maschinen Rechnung zu } \\
\text { tragen }\end{array}$ \\
\hline $\begin{array}{l}\text { Funktionalität, } \\
\text { Zentralität }\end{array}$ & $\begin{array}{l}\text { Die Plattform stellt eine eigenständige, zen- } \\
\text { trale Komponente dar, welche in funktionaler } \\
\text { Hinsicht Mehrwerte (Funktionen, die über die } \\
\text { Funktionen der einzelnen Maschinen hinausge- } \\
\text { hen, z. B. zentrale Auswertungsfunktionen über } \\
\text { den Maschinenpark) zur Verfügung stellt. Für } \\
\text { den Betrieb der Plattform sind keine zusätzli- } \\
\text { chen Elemente auf Soft- und Hardwareebene } \\
\text { notwendig }\end{array}$ & $\begin{array}{l}\text { Die Anforderung stellt si- } \\
\text { cher, dass durch die Platt- } \\
\text { form funktionale Mehrwerte } \\
\text { für den Anwender bereitge- } \\
\text { stellt werden }\end{array}$ \\
\hline $\begin{array}{l}\text { Multi-Device } \\
\text { Fähigkeit }\end{array}$ & $\begin{array}{l}\text { Die Plattform ermöglicht die simultane An- } \\
\text { bindung mehrerer Komponenten und Geräte } \\
\text { (Maschinen und Anlagen) gleichzeitig }\end{array}$ & $\begin{array}{l}\text { Der Aspekt grenzt die Lö- } \\
\text { sungen von reinen Anwen- } \\
\text { dungen zur Statusabfrage } \\
\text { bzw. Steuerung einzelner } \\
\text { Maschinen ab und trägt da- } \\
\text { mit dem Plattformgedanken } \\
\text { Rechnung }\end{array}$ \\
\hline
\end{tabular}

\subsubsection{Qualifizierung}

Um dem spezifischen Erkenntnisinteresse gerecht zu werden, müssen die in die Referenzdatenbank aufgenommenen Fälle entsprechend qualifiziert werden. Einerseits müssen die Referenzprojekte dem Szenario eines Maschinen- und Analgenbauers entsprechen. Andererseits müssen die Kriterien für die Qualifizierung der Referenzfälle im Hinblick auf die Plattform spezifiziert werden. Insbesondere die Mindestanforderungen an ein Projekt und die zugehörige Plattform müssen festgelegt werden. Im Rahmen der Vorarbeiten wurden Qualifizierungskriterien erarbeitet, welche in Tab. 1 zusammengefasst sind.

Nach der Identifikation der in Frage kommenden Referenzfälle müssen diese im Hinblick auf die festgelegten Anforderungen hin qualifiziert werden. Anschließend werden in Vorbereitung auf die Analyse und Auswertung die relevanten Merkmale und Parameter für die Referenzfälle und zugehörigen Plattformen ermittelt. Insge- 
Tab. 2 Qualifizierung der Referenzprojekte

\begin{tabular}{lllllll}
\hline Kriterium & Hersteller & $\begin{array}{l}\text { Konnekti- } \\
\text { vität }\end{array}$ & $\begin{array}{l}\text { Eigenstän- } \\
\text { digkeit }\end{array}$ & $\begin{array}{l}\text { Funktionalität, } \\
\text { Zentralität }\end{array}$ & $\begin{array}{l}\text { Multi-Device } \\
\text { Fähigkeit }\end{array}$ & $\begin{array}{l}\text { Alle } \\
\text { Kriterien }\end{array}$ \\
\hline $\begin{array}{l}\text { Anzahl } \\
\text { erfüllt }\end{array}$ & 153 & 163 & 170 & 159 & 156 & 116 \\
$\begin{array}{l}\text { Anzahl } \\
\text { nicht erfüllt }\end{array}$ & 28 & 18 & 11 & 22 & 25 & 65 \\
\hline
\end{tabular}

samt konnten 181 Fälle ermittelt werden. Diese wurden gemäß den festgelegten Kriterien qualifiziert. Tab. 2 gibt einen Überblick über die Qualifizierung der Referenzfälle. Insgesamt zeigt sich, dass alle Kriterien für die Qualifizierung von Bedeutung sind. Die 116 Fälle, welche alle Kriterien erfüllen, bilden die Grundgesamtheit für die Auswertung und Analyse im weiteren Verlauf.

\section{Analyseergebnisse}

Die Analyse stellt die Auswertung der Informationen aus der Referenzdatenbank dar. Zur Einschätzung der Ergebnisse erfolgt vorab eine deskriptive Charakterisierung der Referenzfälle. Die berücksichtigten Fälle repräsentieren überwiegend Fälle von Großunternehmen (79\%). Obwohl im Rahmen der Recherche die Gruppe der KMU gezielt fokussiert wurde, bilden diese mit $21 \%$ einen vergleichsweisen geringen Anteil. Im Hinblick auf die Domäne innerhalb des Maschinen- und Anlagenbaus stellt die Abb. 3 die Verteilung der Referenzfälle dar.

\subsection{Bereitstellungsstrategien}

Im Hinblick auf die Forschungsfrage 1 sind insbesondere die genutzten Bereitstellungsstrategien der Unternehmen von Relevanz. Die Bereitstellung einer eigenen

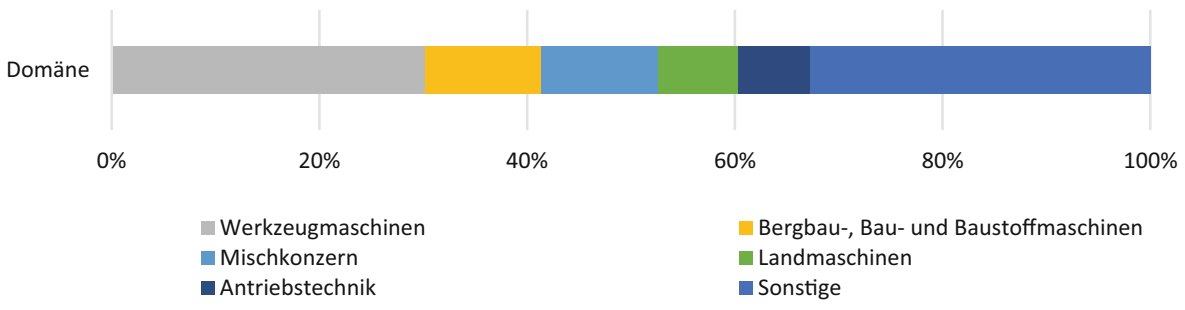

Abb. 3 Charakterisierung der Referenzfälle nach Spezialisierung

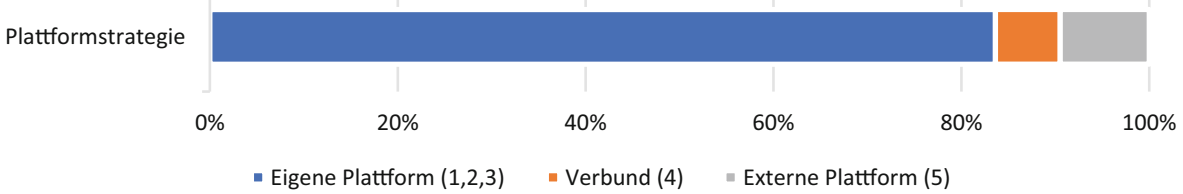

Abb. 4 Verteilung der genutzten Bereitstellungsstrategien 
Plattform (unabhängig vom Anteil der Eigenentwicklung, d.h. Strategien 1, 2, 3) nutzen $83,6 \%$ der Unternehmen. 9,4\% der Unternehmen binden die bereitgestellten Komponenten an eine externe Plattform an (Strategie 5). Die Bereitstellung einer Plattform im Verbund (Strategie 4) nutzen $7 \%$ der Unternehmen. Abb. 4 visualisiert die Verteilung der genutzten Strategien. Es zeigt sich damit eine klare Dominanz hinsichtlich der Bereitstellung einer eigenen Plattform.

\subsection{Einflussfaktoren Bereitstellungsstrategie}

Im Hinblick auf die zweite Forschungsfrage sind insbesondere die Faktoren, welche die Nutzung einer bestimmten Bereitstellungsstrategie begünstigen, von Interesse. Hierfür wurde eine Vielzahl von Faktoren für die Analyse berücksichtigt, welche im Hinblick auf Zusammenhänge untersucht werden.

\subsubsection{Unternehmensgröße}

Eine wesentliche Einflussgröße sind Eigenschaften der Unternehmen, welche die Plattform bereitstellen. Ein Vergleich nach Unternehmensgröße ist insbesondere vor dem Hintergrund der verfügbaren Ressourcen und Know-how von Interesse (siehe Tab. 3). Hierbei zeigt sich, dass die Bereitstellungsstrategien in Abhängigkeit der Unternehmensgröße variieren. Insbesondere im Hinblick auf die Nutzung externer Plattformen wird deutlich, dass lediglich $8 \%$ der Großunternehmen diese Strategie nutzen, dem gegenüber stehen fast ein Fünftel (17\%) der KMU.

\subsubsection{Branchenspezialisierung}

Auch das Produktangebot kann einen Einfluss auf die gewählte Strategie haben, da die Verfügbarkeit von Lösungen und deren Spezifik stark variiert. Während hochspezialisierte Anforderungen dedizierte Lösungen erfordern, so bietet sich in Bereichen mit weitreichenden Gemeinsamkeiten die Nutzung von übergreifenden Lösungen an, um Effizienzvorteile zu realisieren. Beispielsweise werden im spezialisierten Segment der Bergbau-, Bau- und Baustoffmaschinen ausschließlich eigene Plattformen (13) angeboten. Ein hoher Eigenanteil an bereitgestellten Produkten zeigt sich auch im Umfeld der Werkzeugmaschinenanbieter. Hier stellen $91 \%$ eine eigene Plattform zur Verfügung, 6\% nutzen eine externe Plattform und 3\% die Verbundstrategie. Auch bei Werkzeugmaschinen können Spezialanwendungen für die Nutzung der herstellerspezifischen Funktionalität erforderlich sein. Weniger spezifische Lösungen sind z.B. im Bereich der Mischkonzerne gefordert. Mischkonzerne bieten lediglich zu $54 \%$ eine eigene Plattform an. $31 \%$ der Mischkonzerne nutzen externe

Tab. 3 Plattformstrategien nach Firmengröße

\begin{tabular}{llll}
\hline Kriterium & Eigene Plattform & Externe Plattform & Verbund \\
\hline GU & $78(84 \%)$ & $7(8 \%)$ & $7(8 \%)$ \\
KMU & $19(79 \%)$ & $4(17 \%)$ & $1(4 \%)$ \\
Übergreifend & $97(84 \%)$ & $11(9 \%)$ & $8(7 \%)$ \\
\hline
\end{tabular}




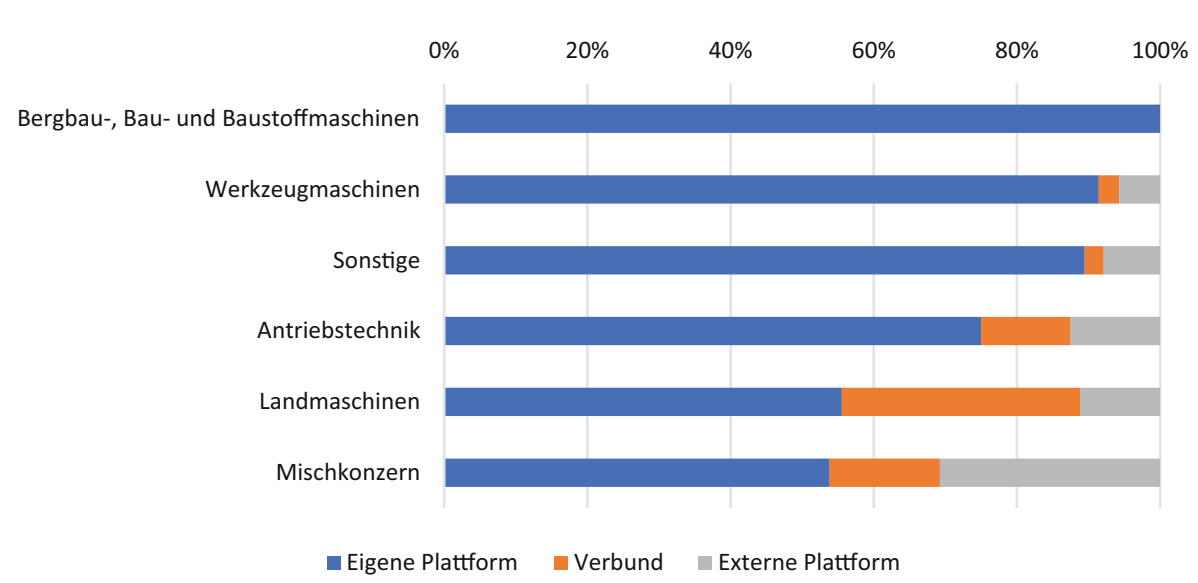

Abb. 5 Plattformstrategie nach Branchenspezialisierung

Plattformen, der Rest (15\%) nutzt die Verbundstrategie. Abb. 5 stellt die genutzte Strategie für die größten Branchenspezialisierungen dar.

\subsubsection{IT-Kompetenzen}

Die IT-Kompetenz in Unternehmen stellt eine zentrale Voraussetzung für das erfolgreiche Angebot und die gezielte Weiterentwicklung einer digitalen Plattform dar. Es wird untersucht, inwieweit die Verfügbarkeit von IT-Kompetenz im Unternehmen, welches durch die Verfügbarkeit einer dedizierten IT-Abteilung operationalisiert wird, einen Einfluss auf die gewählte Bereitstellungstrategie hat. Verfügt ein Unternehmen über eigene IT-Kompetenz, so wird in $85 \%$ der Fälle eine eigene Plattform angeboten. Verfügen Unternehmen über keine eigene IT-Kompetenz wird seltener eine eigene Plattform angeboten. Lediglich etwas mehr als drei Viertel (78\%) bieten dann eine eigene Plattform an, $11 \%$ nutzen eine externe Plattform, $11 \%$ die Verbundstrategie. Weiterhin wird davon ausgegangen, dass der Anteil an Eigenentwicklung an einer Plattform, insofern eigene IT-Kompetenz vorhanden ist, höher ist.

Die Ergebnisse sind ähnlich für die Gesamtgruppe der Fälle und die KMU im Spezifischen. Besitzt ein KMU eigene IT-Kompetenz so bieten diese in $85 \%$ der Fälle eine eigene Plattform an. Hingegen wenn keine IT-Kompetenz zur Verfügung steht, bieten ca. drei Viertel $(73 \%)$ der Unternehmen eine eigene Plattform an. Die übrigen KMU nutzen dann eine externe Plattform (18\%), während die restlichen $9 \%$ die Verbundstrategie nutzen.

\subsubsection{Offenheit gegenüber anderen Herstellern}

Ein weiterer Untersuchungsaspekt stellt die Offenheit der Plattform gegenüber Maschinen anderer Hersteller dar. Aspekte der Plattformoffenheit und -integration sind bereits Bestandteil bisheriger Forschung (Bender 2020; Boudreau 2010; Gawer und Cusumano 2014). Die Bedeutung der Offenheit ist vor dem Hintergrund des zu- 
meist heterogenen Maschinenparks von Anwendern sowie der kapitalintensiven Investitionsgüter im Maschinen- und Anlagenbau von großer Bedeutung. Insgesamt ist ein Drittel der angebotenen Plattformen für die Anbindung von Maschinen anderer Hersteller offen. Die Ergebnisse unterscheiden sich dabei nicht wesentlich zwischen KMU und Großunternehmen. Unterschiede finden sich in Abhängigkeit der Branchenspezialisierung der Anbieter. So findet sich im Bereich der Prozessindustrie, in der verbundene Prozesse hohe Anforderungen an die Durchgängigkeit der Lösungen stellen, eine höhere Offenheit gegenüber anderen Herstellern (z. B. Nahrungsmittelmaschinen, Kälte- und Lufttechnik). Im Unterschied dazu zeichnen sich einige Bereiche durch die ausschließliche Möglichkeit zur Anbindung eigener Maschinen z. B. Verpackungsmaschinen, Papiergewerbe aus.

Im Hinblick auf die zweite Forschungsfrage konnte identifiziert werden, dass die gewählte Bereitstellungsstrategie in Abhängigkeit unterschiedlicher Faktoren variiert. So bieten insbesondere große Unternehmen eigene Plattformen an. Weiterhin bieten insbesondere Unternehmen mit entsprechender IT-Kompetenz eigene Lösungen an. Weiterhin variiert die Bereitstellungsstrategie in Abhängigkeit der Spezifik des Portfolios. Insgesamt konnten wesentliche Unterschiede in Abhängigkeit der Branchenspezialisierung identifiziert werden.

\section{Diskussion}

Die Analyse der Referenzfälle gibt unterschiedliche Ansatzpunkte für Theorie und Praxis. Insgesamt zeigen die Ergebnisse, dass sich die Mehrzahl (84\%) der Unternehmen für die Strategie der eigenen Plattform entscheidet. 9\% der Unternehmen nutzen eine fremde Plattform, wohingegen der Rest die Verbundstrategie nutzt, um Kunden eine Plattform anzubieten. Der insgesamt hohe Anteil an eigenen Plattformen im Maschinen- und Anlagenbau ist bemerkenswert. Gegebenenfalls sind hier die hohe Spezialisierung der Produkte im Vergleich zu anderen Branchen sowie die damit verbundenen individuelle Funktionen ausschlaggebend. Hierzu trägt beispielsweise auch die diffizile Segmentierung des Maschinen- und Anlagenbaus bei. Diese These stützt auch die Berücksichtigung des angebotenen Produktspektrums. Während hochspezifische Branchen (z.B. Bergbau und Baumaschinen) vollständig auf eigene Plattformen setzen, so nutzen insbesondere Mischkonzerne auch verstärkt externe Plattformen und Verbünde. Insgesamt scheint das Produktportfolio einen Einfluss auf die gewählte Strategie zu haben. Ein Vergleich mit anderen Branchen, die sich z. B. durch weniger individuelle Produkte (z. B. Smart Home) auszeichnen, könnte über den Einfluss der Branche Aufschluss geben. Zusammenfassend kann im Hinblick auf die Forschungsfrage 1 die Strategie der eigenen Plattform als vorherrschende, gefolgt von der Anbindung an externe Plattformen und abschließend die Verbundstrategie identifiziert werden.

Die detaillierte Betrachtung der Referenzfälle gibt Aufschluss über Merkmale, die die genutzten Strategien differenzieren. Beim Vergleich nach Größen zeigt sich, dass kleine Unternehmen häufiger externe Plattformen nutzen als große Unternehmen. Aus Ressourcen- und Know-How-Perspektive erscheint dies nachvollziehbar. Während Großunternehmen zumeist über umfangreichere Kapazitäten als 
KMU verfügen, können diese Plattformen, welche selbst umfangreiche Aufwände in Entwicklung, Betrieb und Pflege erfordern, eher bereitstellen als KMU. Dies zeigt sich insbesondere in Kombination mit der Berücksichtigung der vorhanden IT-Kompetenz in den Unternehmen. Unternehmen mit IT-Kompetenz bieten deutlich eher eigene Plattformen an als Unternehmen ohne. Umgekehrt kann das Vorhandensein von IT-Kompetenz als wesentliche Voraussetzung für das Angebot einer Plattform gesehen werden, dies gilt insbesondere für die Strategien 1 (vollständige Eigenentwicklung) und 2 (Nutzung Technologiebausteine), in denen die Unternehmen aktiv an der Konzeption und Entwicklung beteiligt sind. Hierbei bietet die spezifischere Analyse der Bereitstellungsstrategien die Möglichkeit, zusätzliche Zusammenhänge zu identifizieren. In Bezug auf die Forschungsfrage 2 konnte gezeigt werden, dass die gewählte Strategie sich nach unterschiedlichen Merkmalen unterscheidet (u.a. Größe, Produktspektrum, Kompetenzen). Dies bietet einen Ansatzpunkt für zukünftige Forschung. Hierbei sollte evaluiert werden, inwiefern es sich dabei um bewusste Entscheidungen der Unternehmen handelt.

\subsection{Limitationen}

Die Untersuchung unterliegt einigen Einschränkungen. Während die Systematisierung von Bereitstellung generischen Charakter aufweist, so sind die Erkenntnisse der Marktrecherche spezifisch für den Sektor des Maschinen- und Anlagenbaus. Dies schränkt die Generalisierbarkeit der Ergebnisse im Hinblick auf die genutzten Bereitstellungsstrategien ein. Vor Übertragung der Erkenntnisse auf andere Bereiche müssen die strukturellen Ähnlichkeiten der Bereiche berücksichtigt werden.

Die Identifikation möglicher Referenzfälle basiert auf unterschiedlichen Quellen. Wenngleich sich insbesondere im Bereich der KMU-Referenzfälle eine Sättigung ergab (mehrfach gleiche Projekte identifiziert, trotz gezielter Suche keine weiteren ermittelbar), so ist insbesondere im Bereich der Großunternehmen nicht von einer vollständigen Abdeckung des Marktes auszugehen.

\subsection{Zukünftige Forschung}

Weitere Forschungsbemühungen könnten die Detailanalyse von Aspekten sowie die Identifikation von weiteren Einflussfaktoren auf die Wahl der Bereitstellungsstrategien fokussieren. Weiterhin bietet eine verfeinerte Klassifizierung der gewählten Strategie einer eigenen Plattform (aktuell Zusammenfassung von Strategie 1, 2, 3) weitere Ansätze für Untersuchungen. Auf Basis öffentlich verfügbarer Informationen lassen sich diese nicht angemessen differenzieren.

Während die Referenzdatenbank lediglich die Analyse der Ist-Situation über die Referenzprojekte hinweg ermöglicht, könnten in weiteren Vorhaben die Gründe für die Wahl einer bestimmten Strategie im Dialog mit den Unternehmen untersucht werden. Dieser Einblick in die Strategiefestlegung bietet Ansatzpunkte für die Ableitung von Handlungsempfehlungen für Unternehmen. Für Unternehmen stellt sich bei der Einführung einer Plattform neben den unterschiedlichen Bereitstellungsmöglichkeiten insbesondere die Frage, welche der Strategien sich für den gegebenen Kontext bestmöglich zur Erreichung der Ziele eignet. Dies setzt die Bewertung der unter- 


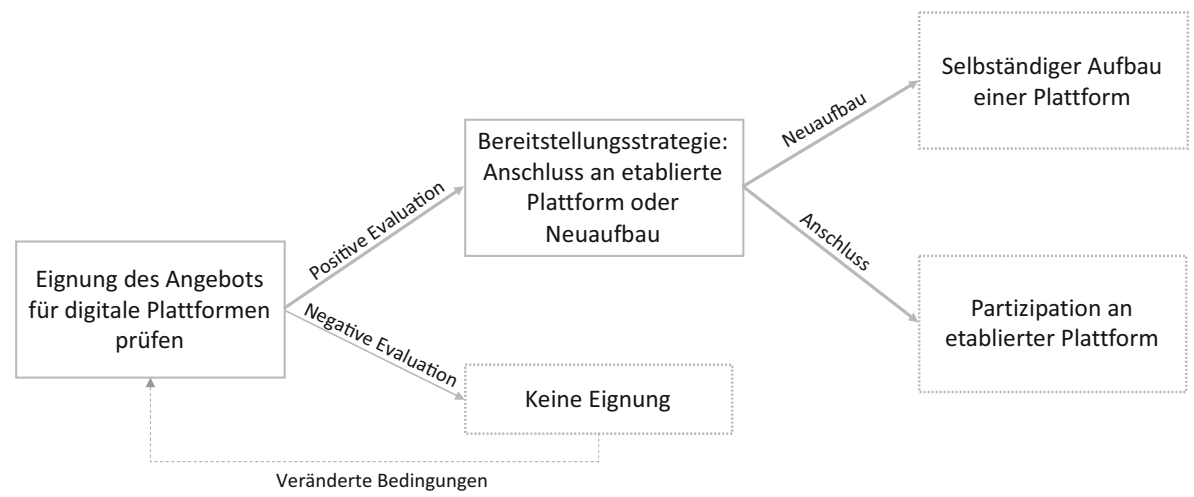

Abb. 6 Entscheidungsmodell zum strategischen Umgang mit digitalen Plattformen

schiedlichen Strategien und damit verbundenen Möglichkeiten und Anforderungen voraus, welche im Dialog mit Unternehmen erarbeitet werden können. Langfristig könnten die Ergebnisse im Rahmen eines Entscheidungsunterstützungstools zusammengeführt werden und so die Erkenntnisse für Unternehmen zugänglich gemacht werden.

Insbesondere im Hinblick auf das Geschäftsmodell ergeben sich weiterreichende Veränderungen für Unternehmen, die eine digitale Plattform bereitstellen. Gerade im produktzentrierten Bereich des Maschinen- und Anlagenbaus bildet die Bereitstellung einer hochverfügbaren IT-Plattform mit Mehrwertdiensten eine Herausforderung. Aufgrund der strategischen Bedeutung sollten mögliche Alternativen sorgfältig bewertet werden. Abb. 6 stellt ein Vorgehensmodell zur Entscheidungsfindung dar. $\mathrm{Zu}$ Beginn des Prozesses sollten Unternehmen bewerten, inwiefern digitale Plattformen für die Erreichung der Geschäftsziele dienlich sind. Im Falle einer positiven Bewertung sollten mögliche Bereitstellungsoptionen betrachtet werden. Hierbei ist insbesondere die Anbindung an eine etablierte Plattform (Strategie 5) von der Möglichkeit zum Aufbau einer eigenen Plattform zu unterscheiden (Strategie 1, 2, 3). Weiterhin besteht die Möglichkeit der Plattformbereitstellung im Verbund (Strategie 4). Zukünftige Forschung könnte die Entscheidungsfindung gemäß des skizzierten Prozesses mittels Tools zur Bewertung der Bereitstellungsalternativen unterstützen. Insbesondere die Gruppe der KMU, welche im Vergleich zu Großunternehmen besonderen Ressourcen- und Know-How-Beschränkungen unterliegt, könnten davon profitieren.

Als weiterer Schritt im Rahmen der Generalisierung bietet sich die Analyse weiterer Domänen an. Somit können Gemeinsamkeiten und Unterschiede in Abhängigkeit der Branche identifiziert werden.

\section{Zusammenfassung}

Digitale Plattformen finden zunehmende Verbreitung in zahlreichen Industriezweigen. Immer mehr Unternehmen sind interessiert, mögliche Potenziale für ihr Ge- 
schäft zu erschließen. Für die Bereitstellung digitaler Plattformen können Unternehmen auf unterschiedliche Strategien zurückgreifen. Hierbei sollten Unternehmen die für ihre Konstellation geeignete Variante systematisch identifizieren, um die angestrebten Ziele zu erreichen. Die geeignete Strategie ist von einer Vielzahl an Faktoren abhängig. Als Grundlage für die Identifikation der geeigneten Strategie bietet dieser Beitrag eine systematische Untersuchung der möglichen Bereitstellungsstrategien für Unternehmen. Neben der theoretischen Systematisierung werden gegenwärtig genutzte Strategien am Beispiel des Maschinen- und Anlagenbaus vorgestellt. Zudem werden spezifische Merkmale, welche die Nutzung einer Strategie beeinflussen, als Ansatzpunkt für einen Strategieformulierungsprozess identifiziert. Im Maschinen- und Anlagenbau ist die Bereitstellung einer eigenen Plattform, insbesondere bei Großunternehmen vorherrschend. Die Strategien von KMU unterschieden sich von Großunternehmen.

Funding Open Access funding provided by Projekt DEAL.

Open Access Dieser Artikel wird unter der Creative Commons Namensnennung 4.0 International Lizenz veröffentlicht, welche die Nutzung, Vervielfältigung, Bearbeitung, Verbreitung und Wiedergabe in jeglichem Medium und Format erlaubt, sofern Sie den/die ursprünglichen Autor(en) und die Quelle ordnungsgemäß nennen, einen Link zur Creative Commons Lizenz beifügen und angeben, ob Änderungen vorgenommen wurden.

Die in diesem Artikel enthaltenen Bilder und sonstiges Drittmaterial unterliegen ebenfalls der genannten Creative Commons Lizenz, sofern sich aus der Abbildungslegende nichts anderes ergibt. Sofern das betreffende Material nicht unter der genannten Creative Commons Lizenz steht und die betreffende Handlung nicht nach gesetzlichen Vorschriften erlaubt ist, ist für die oben aufgeführten Weiterverwendungen des Materials die Einwilligung des jeweiligen Rechteinhabers einzuholen.

Weitere Details zur Lizenz entnehmen Sie bitte der Lizenzinformation auf http://creativecommons.org/ licenses/by/4.0/deed.de.

\section{Literatur}

Bender B (2020) The impact of integration on application success and customer satisfaction in mobile device platforms. Bus Inf Syst Eng. https://doi.org/10.1007/s12599-020-00629-0

Bender B, Grum M, Gronau N, Alfa A, Maharaj B (2019) Design of a worldwide simulation system for distributed cyber-physical production networks. In: 2019 IEEE International Conference on Engineering, Technology and Innovation (ICE/ITMC), S 1-7

Bender B, Habib N, Gronau N (2020) Digitale Plattformen: Strategien für KMU. Wirtschaftsinform Manag (im Druck)

Bollhöfer E, Buschak D, Moll C (2016) Dienstleistungsbasierte Geschäftsmodelle für Industrie 4.0 - aktueller Stand und Potenziale für KMU. In: Multikonferenz Wirtschaftsinformatik, S 1287-1298

Boudreau K (2010) Open platform strategies and innovation: granting access vs. devolving control. Manag Sci 56(10):1849-1872

Demont A, Paulus-Rohmer D (2017) Industrie 4.0-Geschäftsmodelle systematisch entwickeln. In: Schallmo D, Rusnjak A, Anzengruber J, Werani T, Jünger M (Hrsg) Digitale Transformation von Geschäftsmodellen: Grundlagen, Instrumente und Best Practices. Springer, Wiesbaden, S 97-125 https://doi. org/10.1007/978-3-658-12388-8_4

Dremel C, Herterich M (2016) Digitale Cloud-Plattformen als Enabler zur analytischen Nutzung von operativen Produktdaten im Maschinen- und Anlagenbau. HMD 53(5):646-661. https://doi.org/10.1365/ s40702-016-0250-9

Gawer A, Cusumano MA (2014) Industry platforms and ecosystem innovation. J Prod Innov Manag 31(3):417-433 
Gronau N, Grum M, Bender B (2016) Determining the optimal level of autonomy in cyber-physical production systems. In: 2016 IEEE 14th International Conference on Industrial Informatics INDIN, S 1293-1299 https://doi.org/10.1109/INDIN.2016.7819367

Kampker A, Frank J, Jussen P (2017) Digitale Vernetzung im Service. WiSt 46(5):4-11. https://doi.org/10. 15358/0340-1650-2017-5-4

Mobley RK (2002) An introduction to predictive maintenance. Butterworth-Heinemann, Oxford

Oberländer AM, Übelhör J, Häckel B (2019) IIoT-basierte Geschäftsmodellinnovation im Industrie-Kontext: Archetypen und praktische Einblicke. HMD 56(6):1113-1125. https://doi.org/10.1365/s40702019-00570-1

Pflaum A, Schulz E (2018) Auf dem Weg zum digitalen Geschäftsmodell. HMD 55(2):234-251. https:// doi.org/10.1365/s40702-018-0401-2

VDMA (2018) Plattformökonomie im Maschinenbau Herausforderungen - Chancen - Handlungsoptionen. Roland Berger GmbH (Hrsg), München

Weinert N, Plank M, Ullrich A (2017) Metamorphose zur intelligenten und vernetzten Fabrik: Ergebnisse des Verbundforschungsprojekts MetamoFAB. Springer, Berlin 